\title{
Use of Ultrasound for Characterizing Dairy Products
}

\author{
A. S. Dukhin, ${ }^{1}$ P. J. Goetz, ${ }^{1}$ and B. Travers ${ }^{2}$ \\ 1'Dispersion Technology, Inc., 364 Adams Street, New York, NY 10507 \\ ${ }^{2}$ Anatom Technology Ltd., 2803 Shady Grove Court, Baldwin, MD, 21013
}

\section{ABSTRACT}

It has been known for a long time that acoustic measurements offer some unique features for characterizing liquid food products in their intact state, without any preparation or destruction of the product sample. Acoustic characterization can yield information about fat content, droplet size distribution, and kinetics of product variation with time. Furthermore, acoustic methods are very attractive for on-line process control. This paper addresses several questions: Why does ultrasound attenuate when propagating through a heterogeneous system? What properties of dairy products can be extracted from such ultrasound measurements? Which measurement is better for product characterization: attenuation or sound speed? What measurement precision is required to adequately characterize product properties? What frequency range is of most value for determining these product properties?

It is possible to provide answers to many of these questions using experimental data, thereby avoiding, for the moment, any complex mathematical analysis. We present several applications of acoustic spectroscopy for characterizing dairy products, including characterization of the fat content in a wide variety of dairy products; calculation of the fat droplet size distribution in milk without dilution; and calculation of water droplet size in butter, without dilution or melting.

(Key words: ultrasound, droplet size distribution, fat content, process control)

\section{INTRODUCTION}

Ultrasound decreases in intensity and changes phase as it propagates through and interacts with a continuous liquid system. The decrease in intensity is usually referred to as "ultrasound attenuation". The phase is related to the speed of ultrasound propagation through

Received August 11, 2004

Accepted November 8, 2004.

Corresponding author: A. S. Dukhin; e-mail: adukhin@dispersion. com. the particular liquid system. The variation of these 2 properties (attenuation and sound speed) depends in turn on the properties of the liquid system. If we measure the variation of these ultrasound properties then we are also able to extract some information about the properties of the system.

It is an old idea, and there are several commercial instruments that exploit ultrasound to calculate particle size distribution and $\zeta$-potential including such diverse products as paints, pigments, ceramics, clays, inks, and coal slurries (Dukhin and Goetz, 2002). However, only a few ultrasound applications have been concerned with the characterization of food products. One such example is the use of sound speed measurements to measure the alcohol content of beer and other beverages (Anton Paar USA, Ashland, VA).

The use of acoustic characterization in the food industry is now expanding. Ultrasound instruments introduced by Anton Paar USA and Weber Scientific (Hamilton, NJ) specifically target the dairy products market with the aim of providing applications for determining fat content.

Realizing the importance of quantifying food quality and the growing acceptance of acoustic methods, we decided to use our Acoustic Spectrometer DT-100 (Dispersion Technology, Inc., New York, NY) to determine the capabilities and limitations of these methods with regard to characterizing dairy products. This instrument is able to accurately measure attenuation and sound speed at multiple frequencies and for multiple gaps without the need for calibration. Neither of instruments mentioned above has similar capabilities.

In this paper, we describe a set of experiments using several samples of milk and butter. The objective of the study was to demonstrate that acoustic spectroscopy could be used to monitor droplet size distribution and fat content of these samples. In the Appendices, we use our general experience in acoustic spectroscopy to formulate some general conclusions regarding the required measurement precision, the necessary dynamic range of measured properties, and the required knowledge of physical properties of the various food ingredients. 

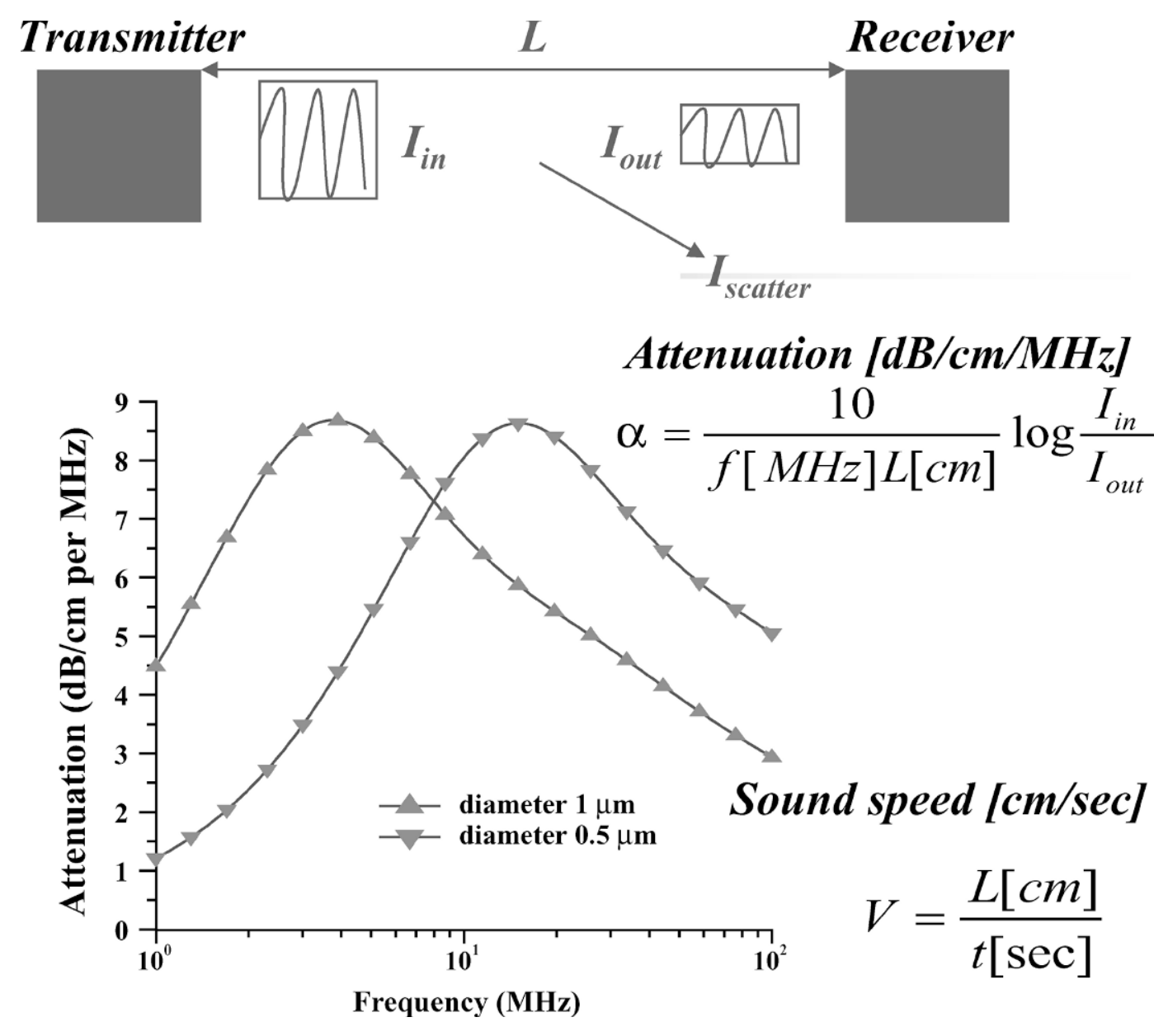

Figure 1. Block scheme of "transmission" acoustic spectrometer.

\section{MATERIALS AND METHODS}

There are 2 generally accepted approaches for measuring the properties of ultrasound as it interacts with a liquid system: interferometric and transmission (Dukhin and Goetz, 2002). The Acoustic Spectrometer DT100 uses the transmission approach to measure attenuation and sound speed at multiple frequencies from 1 to $100 \mathrm{MHz}$ and for multiple gaps between the transmitting and receiving transducers.

Figure 1 illustrates the main principles of the transmission approach. A piezo-electric transducer converts an input electrical tone burst to an ultrasound pulse of a certain frequency and intensity and launches it into the sample. The intensity of this pulse decays as it passes through the sample due to interaction with the fluid. A second piezo-electric transducer converts this weakened acoustic pulse back to an electric pulse and sends it to the electronics for comparison with the initial input pulse. The total loss and time delay from the input to output transducer for each frequency and gap can be considered the raw data from which further interpretation is made. This interpretation is done in 2 steps, involving successively more model assumptions and knowledge of the properties of the dispersion ingredients.

The first step is purely phenomenological in that it requires no assumptions or model of the disperse system under investigation. This step includes calculation of either the acoustic properties (attenuation and sound speed) or rheological properties $\left(G^{\prime}\right.$ and $\left.G^{\prime \prime}\right)$ of the system. Figure 2 describes the relationship between the raw data (output intensity and time of flight) and the desired acoustic properties (attenuation and sound speed) and rheological properties ( $\mathrm{G}^{\prime}$ and $\left.\mathrm{G}^{\prime \prime}\right)$.

It should be noted that the rheological properties are related to high frequency longitudinal excitation of the sample on a microscopic scale, in which the stress is normal to the strain. As such, they differ substantially in nature from the more traditional low frequency shear properties measured on a macroscopic scale in which 
Raw data: - intensity of ultrasound pulse after propagation $\boldsymbol{I}_{\text {out }}$;

- time of the pulse flight $t$; phase of the sound after propagation

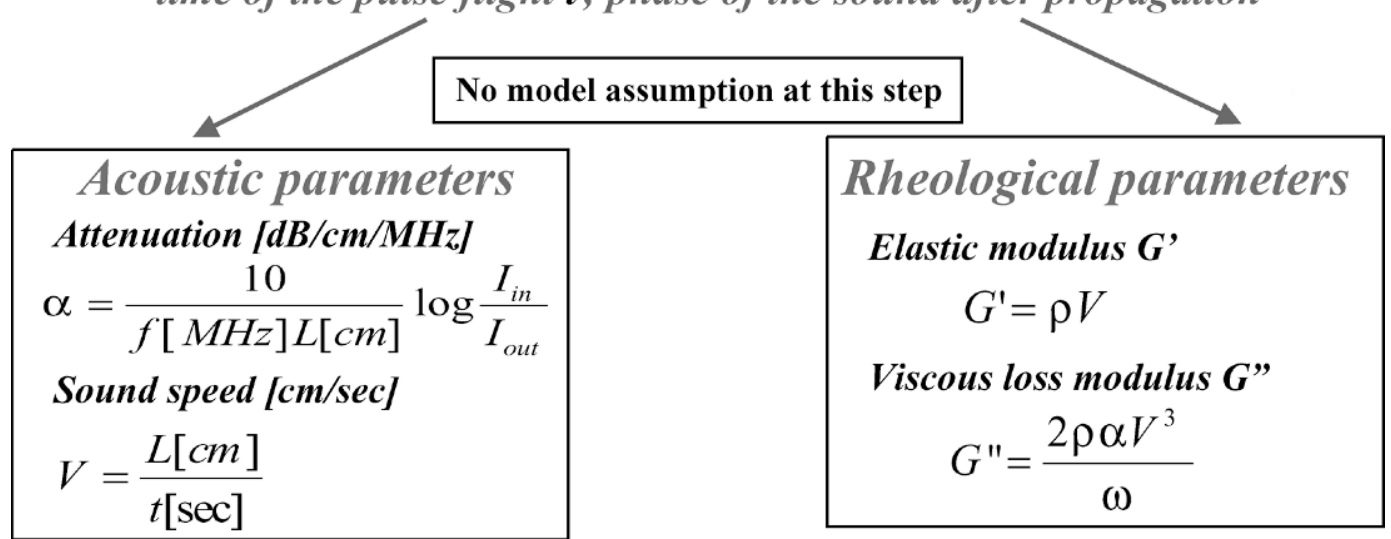

\begin{tabular}{|l|l|}
\hline $\begin{array}{l}\text { Model assumption: Collection } \\
\text { of spherical particles in liquid }\end{array}$ \\
\hline Particle size distribution \\
\hline $\begin{array}{l}\text { Model assumption: If particles } \\
\text { are connected with specific forces }\end{array}$ \\
\hline
\end{tabular}

Hookean coefficient

Figure 2. Various models for interpreting acoustic experimental data.

the stress is tangential to the applied strain. The higher frequency acoustic measurements lead to much higher viscosity values than obtained for the more commonly measured low frequency shear data. However, these acoustically derived rheological parameters can be used for empirical correlations with observed processes, much as traditional low frequency shear wave viscometry is used for this purpose.

The second step in the interpretation of the raw data requires that we adopt a model to describe the real dispersed system as well as some knowledge of certain physical properties of the ingredients of the disperse system. If the adopted model is an adequate representation of the real-world system, and if we have sufficient knowledge of certain physical properties of the system, then the acoustic properties calculated in the first step can be used to compute the particle size distribution, and perhaps other important properties of the sample (e.g., specific forces binding particles together in structured dispersions). For instance, we can calculate size distribution of the fat droplets in milk because we can adequately model milk as a collection of more-or-less spherical fat droplets in a water solution of proteins and sugars, and we know (or can measure) the relevant physical properties of the constituent ingredients. We will show that we are able to perform such calculations of particle size not only in relatively dilute systems such as milk, but also in concentrated systems such as butter.

Such calculation of particle size requires a theory of sound propagation in heterogeneous systems. A general overview of this theory can be found in Dukhin and Goetz (2002). We will simply state here that an adequate theory does exist, even for very concentrated systems up to $50 \%$ by volume and for systems that have some interconnecting structure or more than one dispersed phase. Appendix 1 gives a short description of the main features of this acoustic theory that are relevant to dairy products.

\section{Experimental Procedures}

We used dairy products from 2 sources. One source was a local supermarket where we purchased various milks and butters. The nutritional labels on these product containers defined the product composition, including the fat, protein, and sugar content. We used some of these data in our analysis. In addition, we obtained 


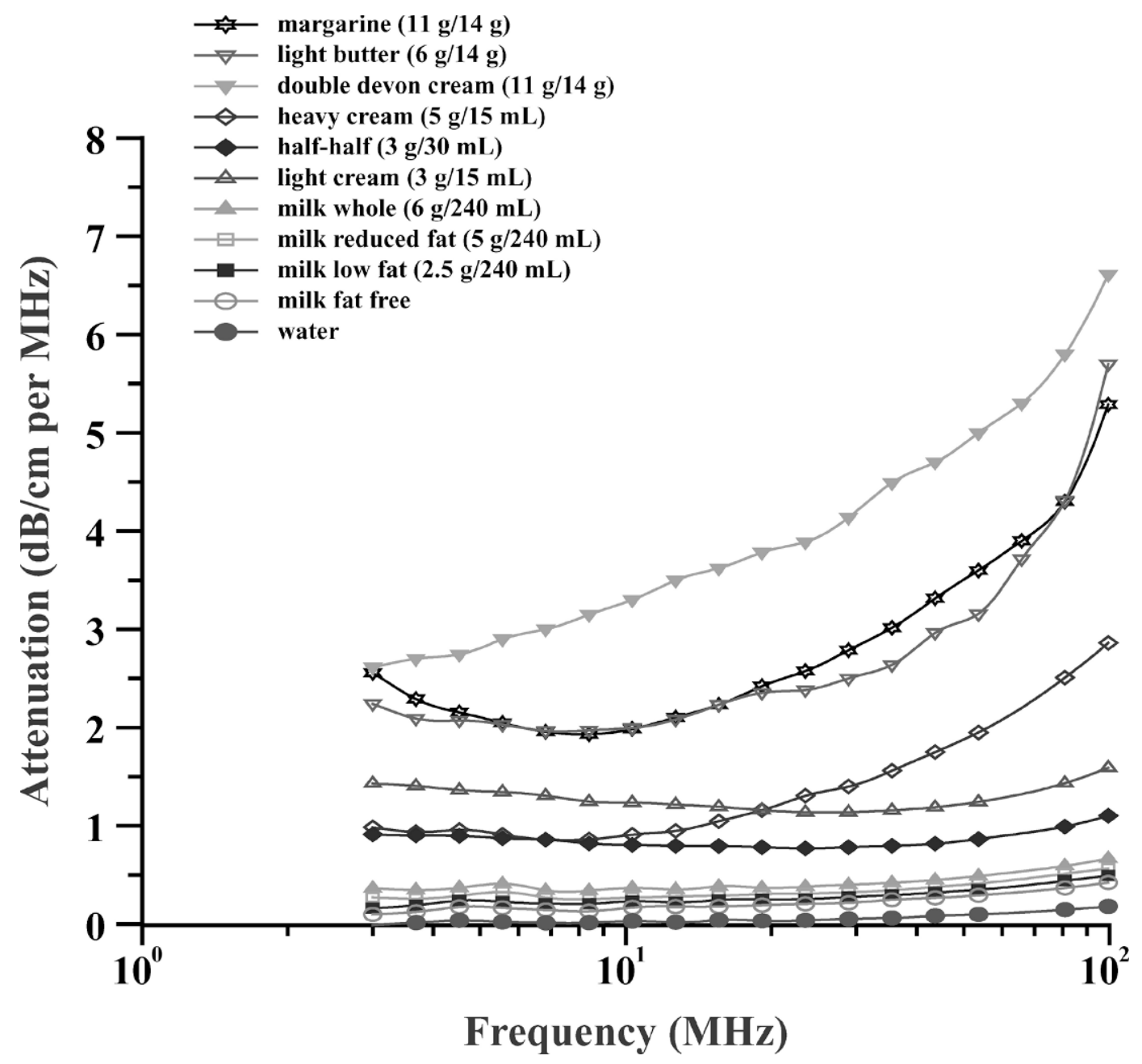

Figure 3. Attenuation of various dairy products.

2 well-defined samples of whole milk from Carmen Moraru at the Department of Food Science, Cornell University (Ithaca, NY). One of these samples was homogenized, and both samples had the same composition: $3.9 \%$ fat, $3.25 \%$ protein, and $4.6 \%$ lactose. These 2 samples allowed us to verify the accuracy of the particle size calculation procedure.

No preparation was involved with any of the samples. Milk and butter samples were measured without dilution. The milk was poured into the DT-100 sample chamber. The required sample volume was 20 to 100 $\mathrm{mL}$ depending on the instrument setup. No mixing or pumping is required for measuring the attenuation of stable products that do not settle. The DT-100 does have a built-in magnetic stirrer, which can be used for mixing an unstable sample. For example, the stirrer was used for measuring the sound speed of a milk sample during a spoiling process, where the resultant ag- glomerates would otherwise settle without such continuous agitation.

Butter was allowed to reach room temperature, at which point it was sufficiently soft to be pushed into the chamber. Measurements of butter must be performed in a mode in which the gap between the transmitting and receiving transducers closes during the measurement. During this measurement, the butter was squeezed between the transducers and extruded from the gap between them. (This is opposite to the normal mode of operation for more liquid samples, in which the gap opens rather than closes during the measurement.)

We obtained the milk fat fraction from a quantity of unsalted butter by heating it and withdrawing the pure milk fat middle phase from the clarified butter phase separation. This separation procedure was necessary to obtain a suitable sample for measuring the intrinsic acoustic properties of the fat. 


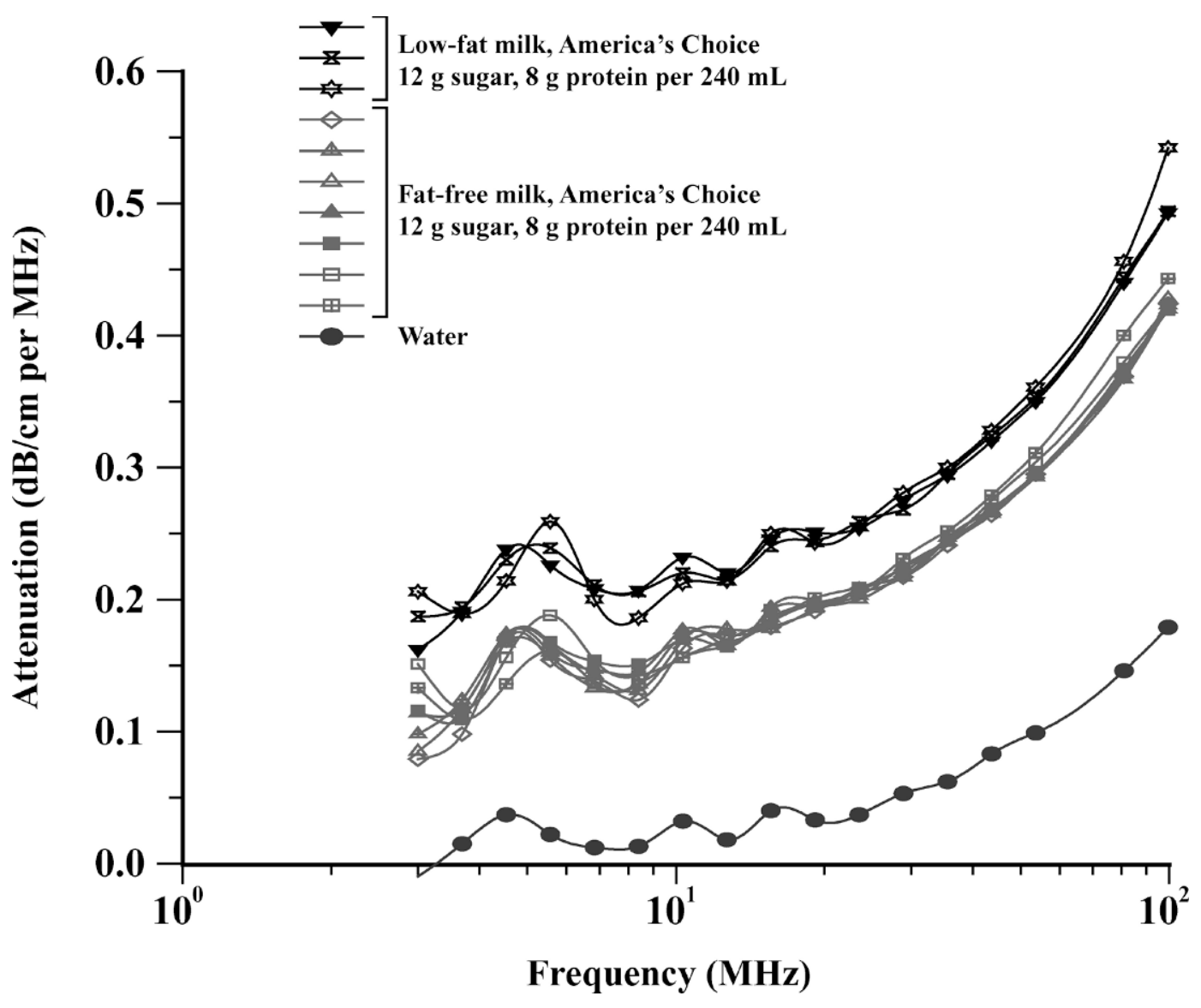

Figure 4. Precision test with low-fat and fat-free milks.

\section{Experimental Data}

The measured attenuation spectra of the milk and butter samples, the result of the first step calculations, are shown in Figures 3, 4, and 5.

Figure 3 presents the attenuation spectra for a variety of dairy products. The legend defines the type of product, the relative fat content according to the manufacturer's label, and the date and time of each measurement. Figure 3 shows one typical curve for each product, but each product was measured several times to test reproducibility. For example, Figure 4 shows multiple measurements for 2 of the low-fat products, as well as a spectrum of plain water for comparison.

Figure 5 shows the attenuation spectra for the 2 milk samples from Cornell University. The samples are identical in composition but different in terms of homogenization (and therefore one would also expect different in the fat droplet size distribution). The distinctive difference in the attenuation spectra between these 2 milk samples is itself proof that acoustic spectroscopy can be used to characterize differences in the particle size of dairy products. The attenuation spectrum within the frequency range from 3 to $100 \mathrm{MHz}$ contains information on the droplet size distribution and we will show how this size information can be extracted.

The calculation of the droplet size distribution and fat content requires certain information about the milk fat properties. Therefore, we measured attenuation spectra of the pure milk fat, which are shown in Figure 6 . We found that the acoustic properties of the milk fat are temperature-dependent. In order to incorporate temperature dependence into the size calculation, we measured the intrinsic attenuation of the fat as a function of temperature. The intrinsic attenuation decreases with temperature in an almost linear manner, as illustrated in Figure 7.

\section{RESULTS AND DISCUSSION}

The experimental data presented in the previous section contain information for calculating droplet size distribution and fat content of these samples. The theoretical procedure for making this calculation is described in Dukhin and Goetz (2002). A short description of the 


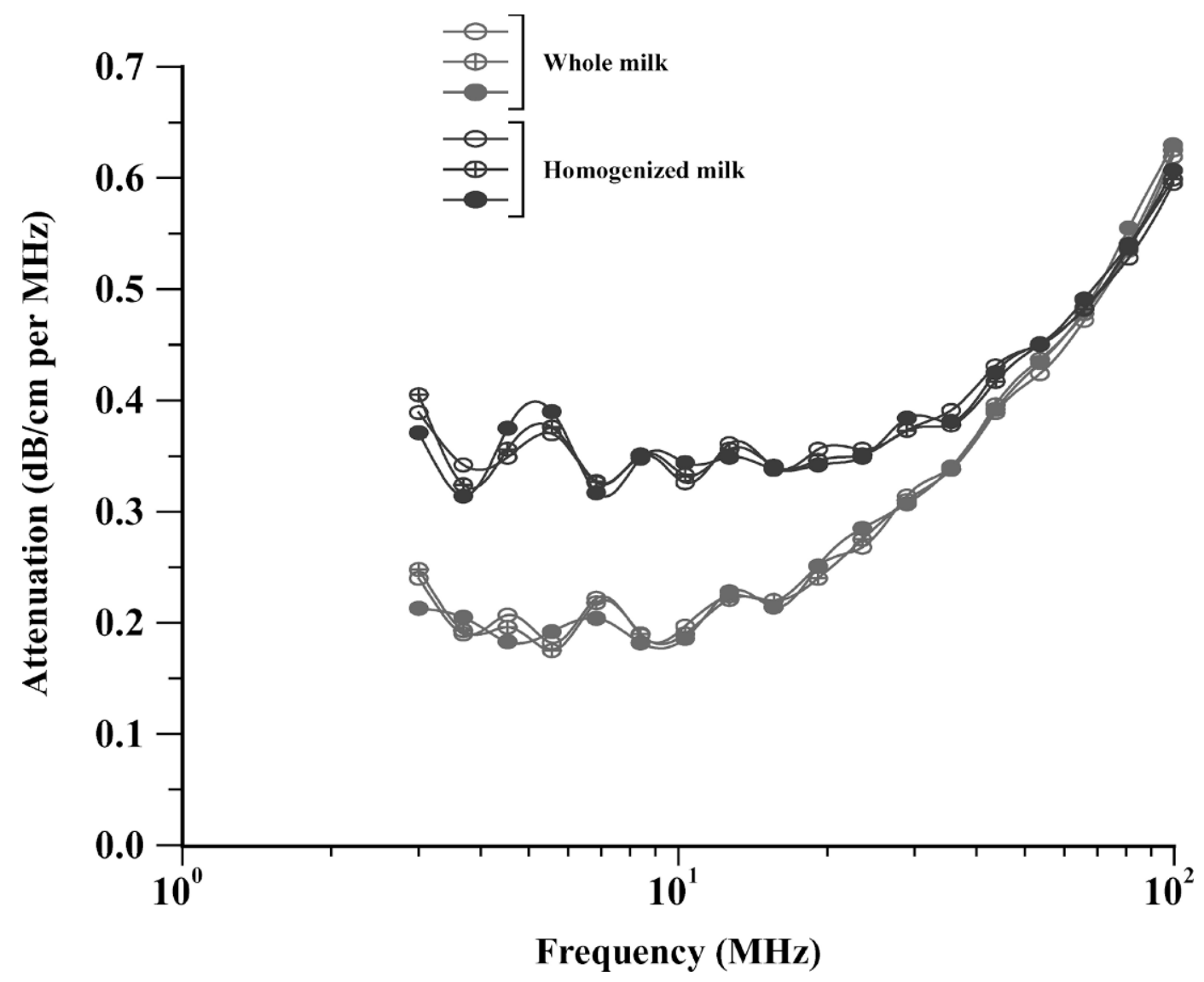

Figure 5. Attenuation spectra of the whole milk and homogenized milk.

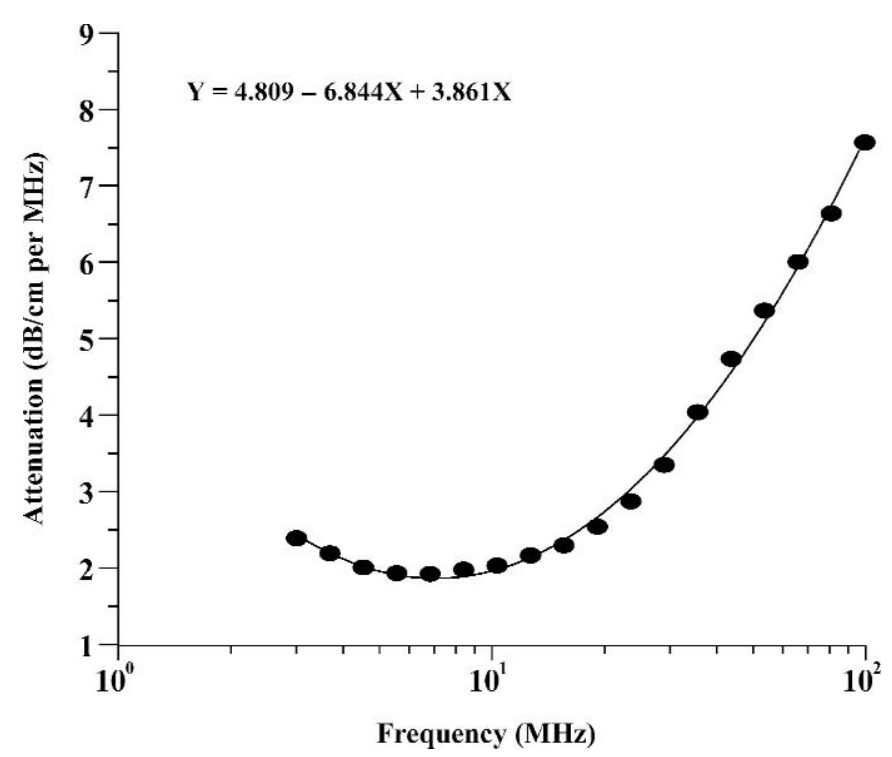

Figure 6. Intrinsic attenuation of the milk fat at room temperature.

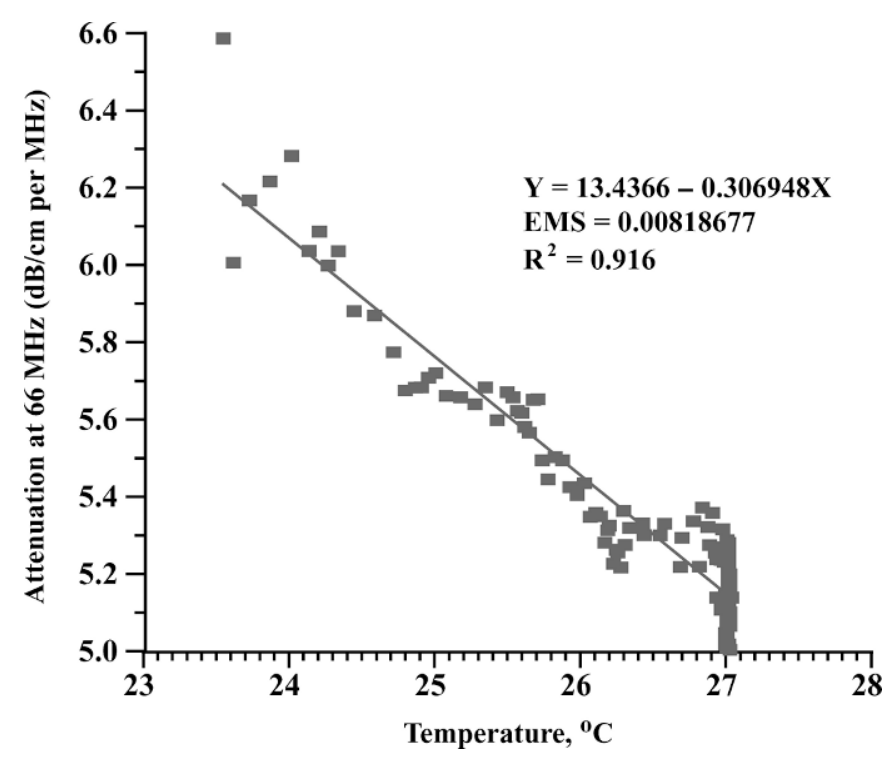

Figure 7. Temperature dependence of the milk fat attenuation at $66 \mathrm{MHz}$. 
Table 1. Thermodynamic properties of the milk fat and water phase around room temperature.

\begin{tabular}{lllll}
\hline & $\begin{array}{l}\text { Thermal conductivity } \\
\left(\mathrm{J} / \mathrm{m} \mathrm{s} \mathrm{K}^{\circ}\right)\end{array}$ & $\begin{array}{l}\text { Heat capacity } \\
(\mathrm{J} / \mathrm{kg} \mathrm{K})^{\circ}\end{array}$ & $\begin{array}{l}\text { Thermal expansion } \\
\left(10^{-4} 1 / \mathrm{K}^{\circ}\right)\end{array}$ & $\begin{array}{l}\text { Attenuation }(\mathrm{dB} / \mathrm{cm} \mathrm{per} \mathrm{MHz})- \\
\text { frequency }[\mathrm{MHz}] \text { function. }\end{array}$ \\
\hline Water & 0.61 & 4.18 & 2.07 & $\alpha=4.809-6.844 \omega+3.861 \omega^{2}+0.147 \omega^{3}$ \\
Milk fat & 0.17 & $2.1\left(\right.$ at $\left.40^{\circ} \mathrm{C}\right)$ & 6.2 & $\alpha=0.002 \omega$ \\
\hline
\end{tabular}

various mechanisms of ultrasound attenuation is provided in Appendix 2.

The calculation of the droplet size distribution requires some knowledge of the physical properties for the each phase. The particular set of required properties depends strongly on the nature of the system. Dairy products are emulsions-oil-in-water for milks and water-in-oil for butters. The densities of the fat and water are very similar. This lack of density contrast between the particles and the surrounding media makes the contribution of the viscous dissipation mechanism to the total attenuation rather insignificant. In addition, the droplet size of these systems usually does not exceed $10 \mu \mathrm{m}$. In combination with the low-density contrast, this makes any contribution to the total attenuation from scattering effects insignificant as well.

This discussion leads to the conclusion that the ultrasound attenuation in dairy products occurs mostly due to thermal dissipation and the intrinsic attenuation of the water and fat phases. Using the terminology from our book (Dukhin and Goetz, 2002), we can describe these systems as having soft particles. Therefore, we need a set of 3 thermodynamic properties for each phase, namely the thermal conductivity $\left(\tau ; \mathrm{J} / \mathrm{m} \mathrm{s} \mathrm{K}^{\circ}\right)$, heat capacity $\left(\mathrm{Cp} ; \mathrm{J} / \mathrm{kg} \mathrm{K} \mathrm{K}^{\circ}\right)$, and thermal expansion $(\beta$; $10^{-4} 1 / \mathrm{K}^{\circ}$ ). In addition, we need the intrinsic attenuation of the fat and water phase, recognizing that the attenuation $\alpha$ of the fat might also be frequency dependent. The thermodynamic properties and the intrinsic attenuation of the milk fat and water are summarized in Table 1.

It is important to mention here that we neglect, for now, the influence of proteins, sugar, and other additives on the properties of the water phase. For the moment, we assume that the acoustic properties of this liquid medium, a complex mixture of water, proteins, and sugars, can be approximated by plain water. This assumption can be tested in subsequent experiments. In this study, we also neglected any temperature dependence of the 3 thermodynamic properties. These assumptions create a small amount of uncertainty in the calculated droplet size distribution.

\section{Particle Sizing in Milk}

Milk is an obvious candidate for verifying the particle sizing capability of ultrasound-based techniques. Ho- mogenization leads to a substantial reduction of the fat droplet size. The attenuation spectra presented in Figure 5 reflect this change. Figure 8 shows the droplet size distributions calculated from these attenuation spectra using the input parameters presented in Table 1.

It is interesting to compare the individual contributions of each attenuation mechanism to the total attenuation. Figure 9 shows the predicted intrinsic, thermal, and total attenuation for the size distribution computed for the unhomogenized milk sample previously shown in Figure 8. It is seen that at low frequency the thermal and intrinsic attenuation are comparable. At high frequency, the intrinsic attenuation is dominant. This observation has a profound effect for fat content calculation, as we will discuss shortly. We can conclude that the attenuation in the low frequency range below 10 $\mathrm{MHz}$ is the most sensitive to changes in particle size resulting from homogenization.

\section{Particle Sizing in Butter}

An interesting feature of the acoustic attenuation procedure is that it allows characterization of the drop-

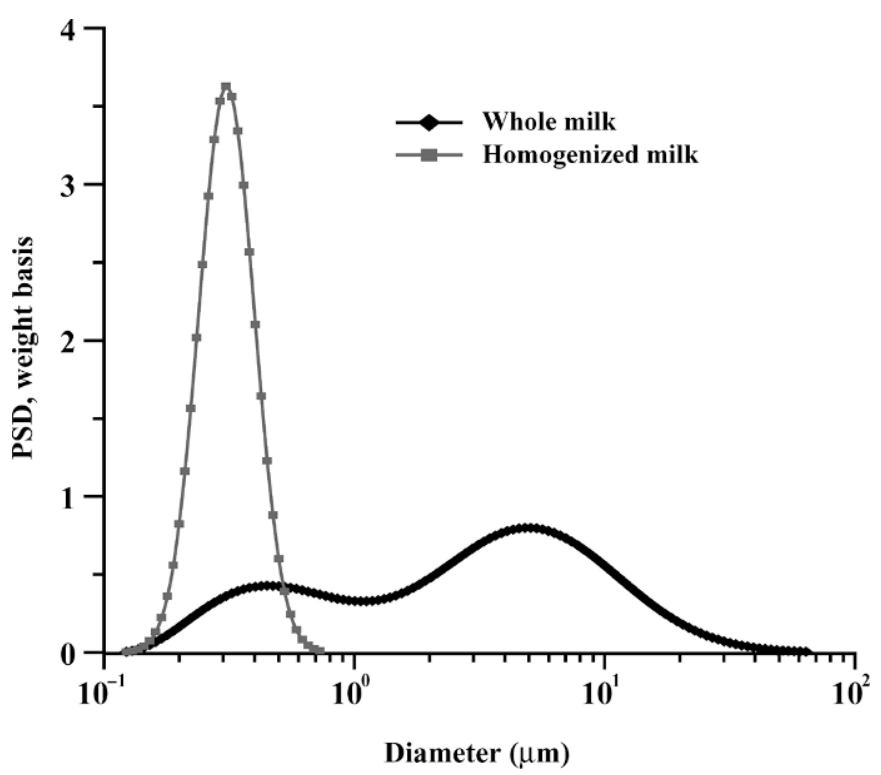

Figure 8. Particle size distribution (PSD) of the fat in whole and homogenized milks. 

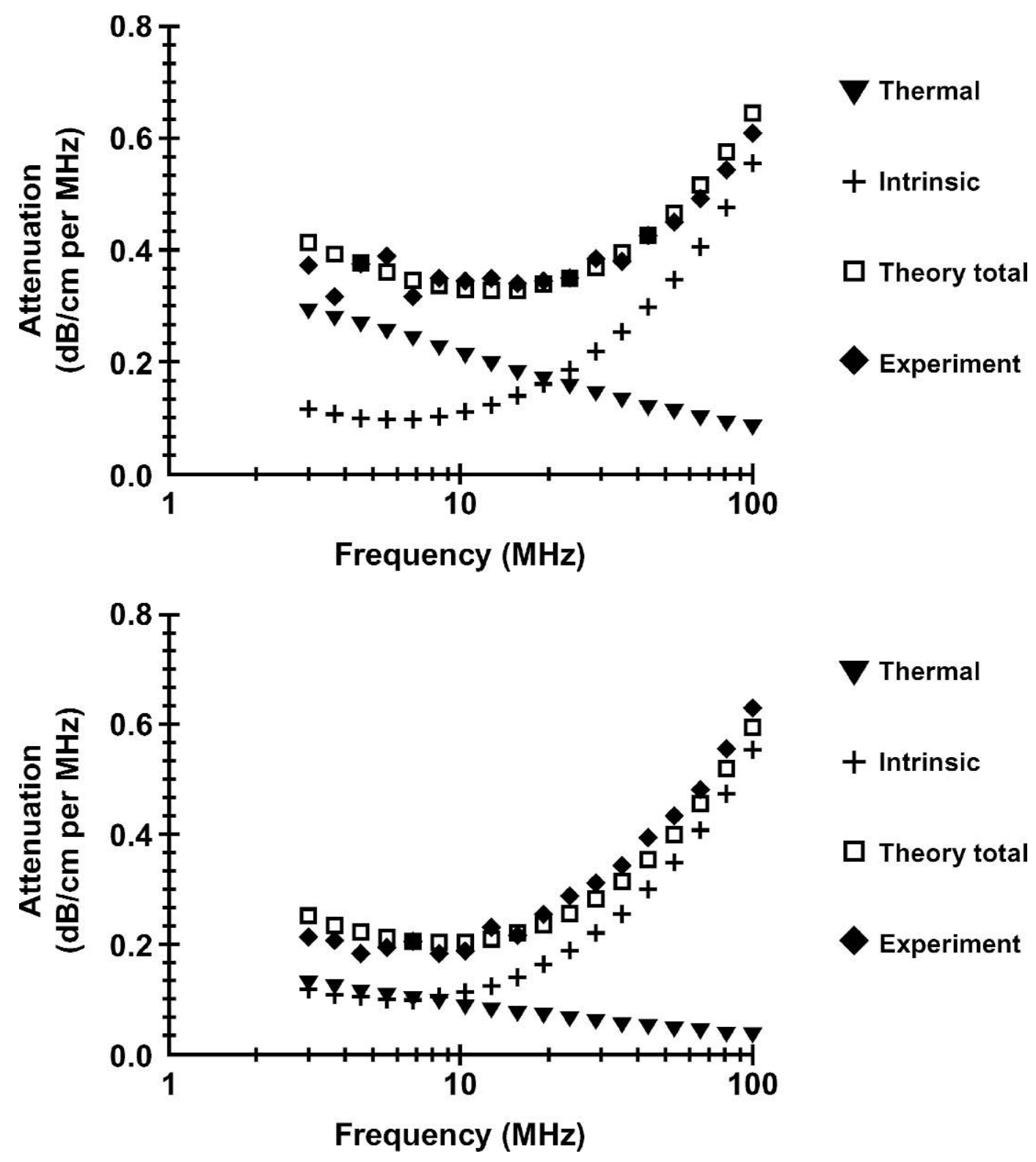

Figure 9. Experimental and theoretical attenuations for whole (lower panel) and homogenized milk (top panel).

let size distribution even in semi-solid systems such as butter, with no dilution or melting. This is possible largely because the thermal attenuation effect is thermodynamic in nature and does not require relative droplet-liquid motion.

Figure 10 shows the computed size of water droplets in light butter. The second graph in Figure 10 presents the theoretical attenuations showing the individual contributions from intrinsic and thermal effects. The intrinsic contribution is much larger for butter than for milk because the intrinsic attenuation of fat is larger than water and butter has a larger fat content. The intrinsic attenuation is the dominant contributor to the total attenuation spectrum at high frequencies, even more so than in the case of milks that have a lower fat content.

These calculations use the same thermodynamic properties for the fat as in the case of the milks discussed earlier.

\section{Determination of Fat Content}

Fat content is a very important quality control parameter for dairy products. The attenuation measurement 

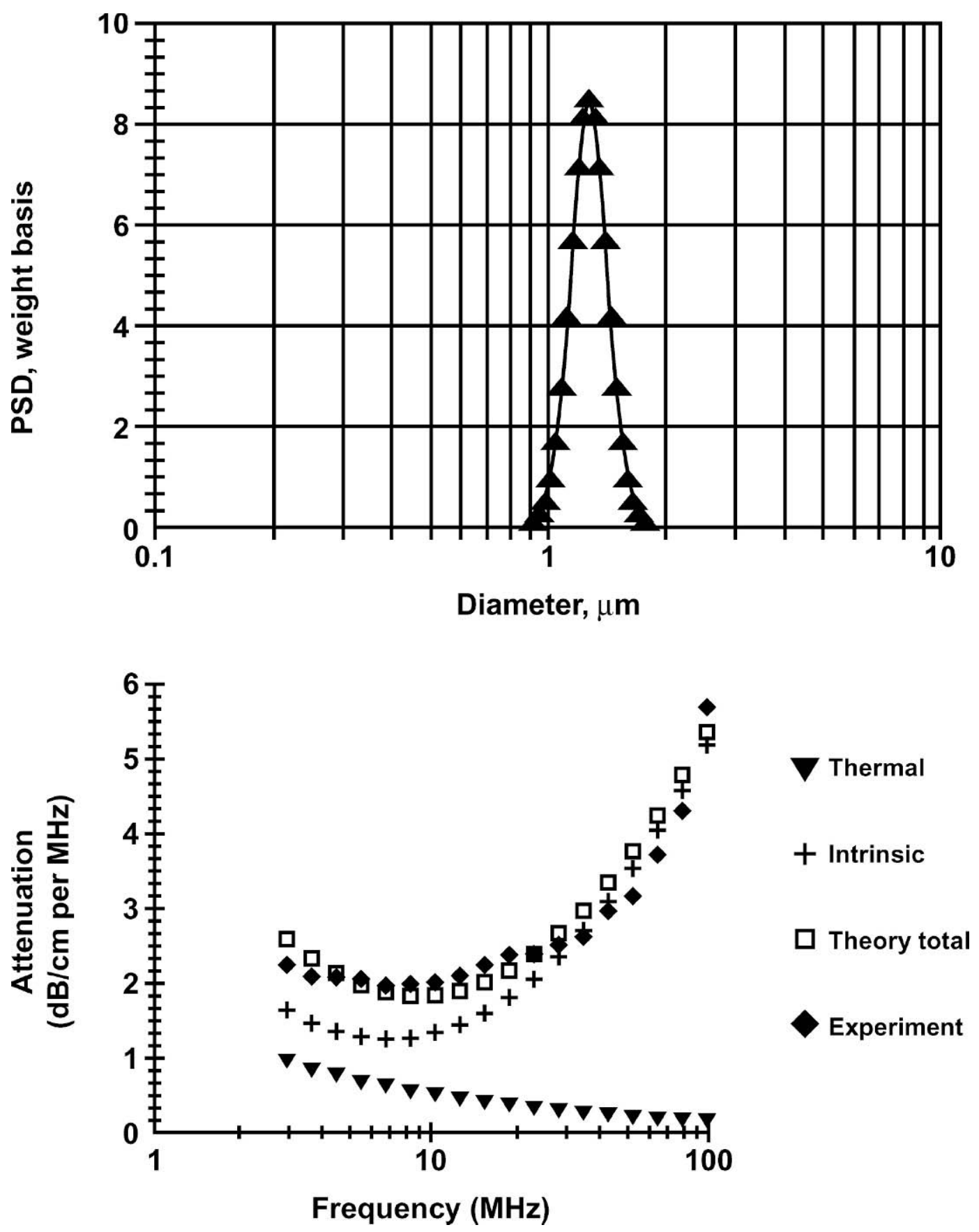

Figure 10. Particle size distribution (PSD) of the water droplets in light butter and corresponding attenuation spectra.

offers a simple way to characterize fat content with no special treatment of the sample. Figure 3 shows the attenuation spectra of various dairy products. It is clear that attenuation increases with increasing fat content. This dependence can be used to calculate fat content.

In previous sections, we showed that the total ultrasound attenuation consists primarily of thermal and intrinsic attenuation. Thermal attenuation is droplet size-dependent, whereas intrinsic attenuation is not. It is clear that the dependence of attenuation on droplet size complicates the characterization of fat content. We can minimize this complexity by using the attenuation at a frequency range where the intrinsic effects are dominant. Figures 9 and 10 indicate that the intrinsic effects are dominant in the high frequency range, above $40 \mathrm{MHz}$. 

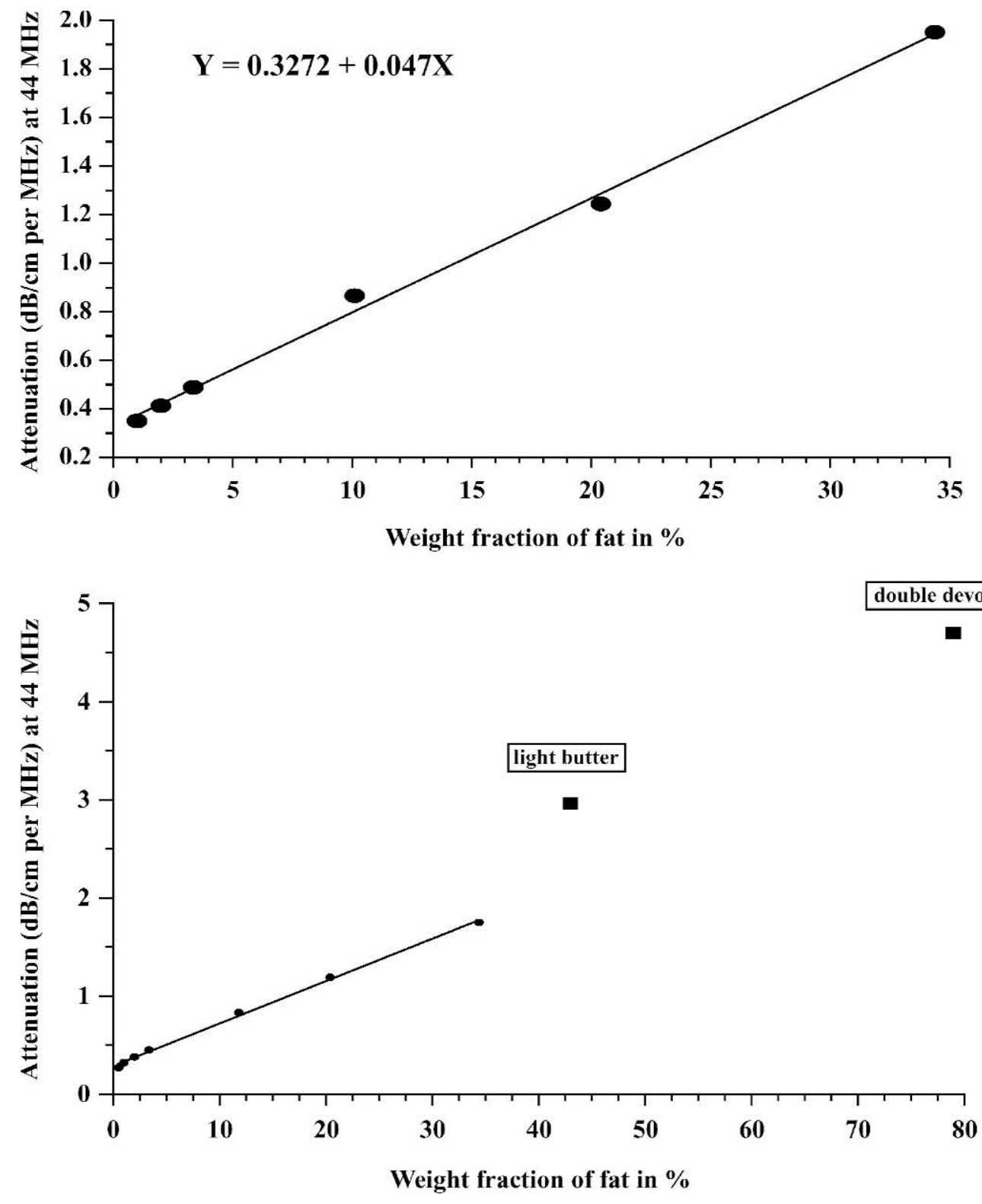

Figure 11. Attenuation of the oil-in-water dairy products at the single frequency of $44 \mathrm{MHz}$ versus fat content.

If we abstract the data for a single frequency (e.g., $44 \mathrm{MHz}$ ) from Figure 3, we can construct a plot of the total measured attenuation at this frequency vs. the fat weight fraction, as illustrated in Figure 11. It is seen that there is a good linear correlation between the attenuation at this frequency and the fat content specified by the producer of the product. This correlation suggests that this single frequency measurement might be a simple and direct way of determining fat content.

We might expect high reproducibility of this procedure because the attenuation spectra measured for sev- eral different brands of the same dairy product are practically the same, as illustrated in Figure 12 for 2 different half-and-half milks.

\section{CONCLUSIONS}

The measured acoustic spectra contain information on droplet size distribution and fat content of dairy products. Attenuation at frequencies below $50 \mathrm{MHz}$ depends substantially on droplet size and can be used to calculate droplet size. The attenuation at frequencies above $50 \mathrm{MHz}$ is dominated by intrinsic attenuation, 


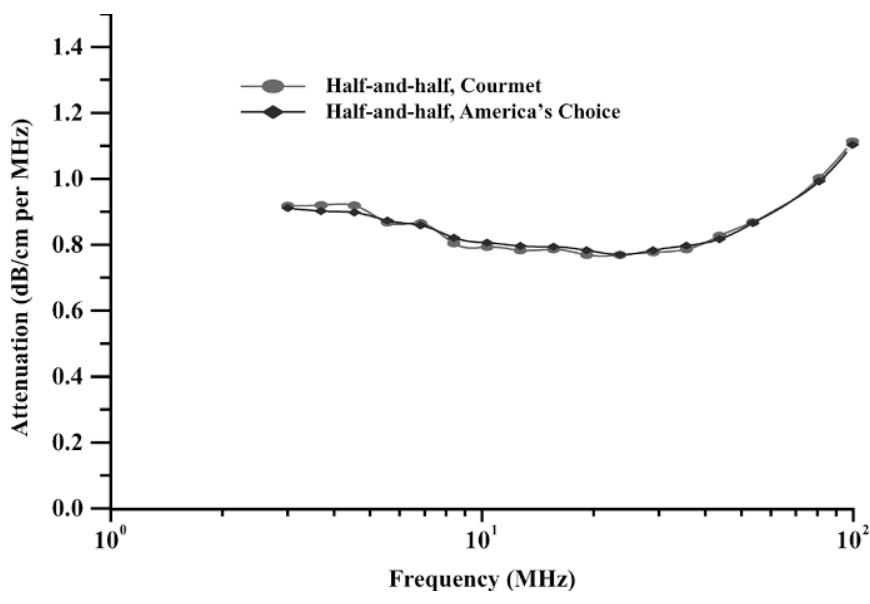

Figure 12. Attenuation of half-and-half milk from 2 different brands.

which is independent of particle size and therefore most attractive for determining fat content.

Calculation of the droplet size distribution requires some knowledge of the properties of the disperse phase and media. These properties include the thermodynamic properties of the fat and water phases and their frequency- and temperature-dependent intrinsic attenuations. We determined these parameters using independent experiments, information from Cornell University, and the literature. A knowledge of these properties allowed us to obtain reasonable droplet size distributions for whole milk, homogenized and unhomogenized milks, as well as light butter. No dilution, melting, or other sample preparation was required to measure any of the milk or butter samples.

We also showed that there is a substantially linear correlation between the high frequency attenuation and fat content for various commercial dairy products. This opens an opportunity to use a single frequency attenuation measurement for characterizing fat content. We derived a number of conclusions regarding acoustic attenuation measurement from our long experience dealing with this characterization technique.

The acoustic attenuation frequency spectrum is a better property for characterizing the dispersed phase and phase composition, whereas sound speed is better for characterizing chemical composition or chemical reactions that occur on a molecular level. Sound speed is much more sensitive to temperature than attenuation. Attenuation of typical food products varies from 0.01 to $20 \mathrm{~dB} / \mathrm{cm}$ per $\mathrm{MHz}$ within the frequency range 1 to $100 \mathrm{MHz}$. It is very much frequency-dependent. The sound speed of typical food products varies from 1000 to $2000 \mathrm{~m} / \mathrm{s}$, and is not very frequency-dependent. Attenuation measurement requires a precision of $0.01 \mathrm{~dB} /$ $\mathrm{cm}$ per $\mathrm{MHz}$. Sound speed measurement requires precision of $1 \mathrm{~cm} / \mathrm{s}$ with temperature stabilization to about $0.1^{\circ} \mathrm{C}$

\section{ACKNOWLEDGMENT}

We would like to express our gratitude to Carmen Moraru from Cornell University (Ithaca, NY) for providing us with samples, useful information on dairy products, and very productive discussion.

\section{REFERENCES}

Dukhin, A. S., and P. J. Goetz. 2002. Ultrasound for characterizing colloids. Particle sizing, Zeta potential, Rheology. Elsevier, the Netherlands.

Isakovich, M. A. 1948. On propagation of sound through emulsions. Zh. Exsp. Teor. Fiz. 18:905-912. (In Russian)

McClements, D. J. 1991. Ultrasonic characterization of emulsions and suspensions. Adv. Colloid Interface. Sci. 37:33-72.

Morse, P. M., and K. Uno Ingard. 1986. Theoretical Acoustics. Princeton University Press, Princeton, NJ.

Rayleigh, J. W. 1896. The Theory of Sound. Vol. 2. 2nd ed. Macmillan and Co., New York, NY.

Stokes, G. G. 1848. On a difficulty in the Theory of Sound. Phil. Mag. $33: 349-356$.

\section{Appendix 1. General Features of Acoustic Measurement}

In this Appendix we present some requirements for the acoustic characterization of dairy products based on our experience in dealing with a broad spectrum of applications.

\section{Attenuation or Sound Speed?}

As we mentioned above, there are 2 measurable acoustic parameters: attenuation and sound speed. The question arises as to which is better for characterizing particular food properties in a given product. There is some disagreement in the general scientific literature. For instance, a well-known scientist in the field of ultrasound characterization of food products stresses the importance of sound speed (McClements, 1991). In contrast, our group relies mostly on attenuation measurements. It is clear that this question deserves some special consideration.

We have performed several simple tests for answering this question. First, we measured the sound speed of water at various concentrations of simple chemicals. As illustrated in Figure 13, sound speed is very sensitive to the chemical composition, changing roughly 100 $\mathrm{m} / \mathrm{s}$ at a concentration of $1 \mathrm{~mol} / \mathrm{L}$ of a simple salt. Keeping in mind that the precision of sound speed measurements is approximately $1 \mathrm{~cm} / \mathrm{s}$ (Figure 14), we can con- 


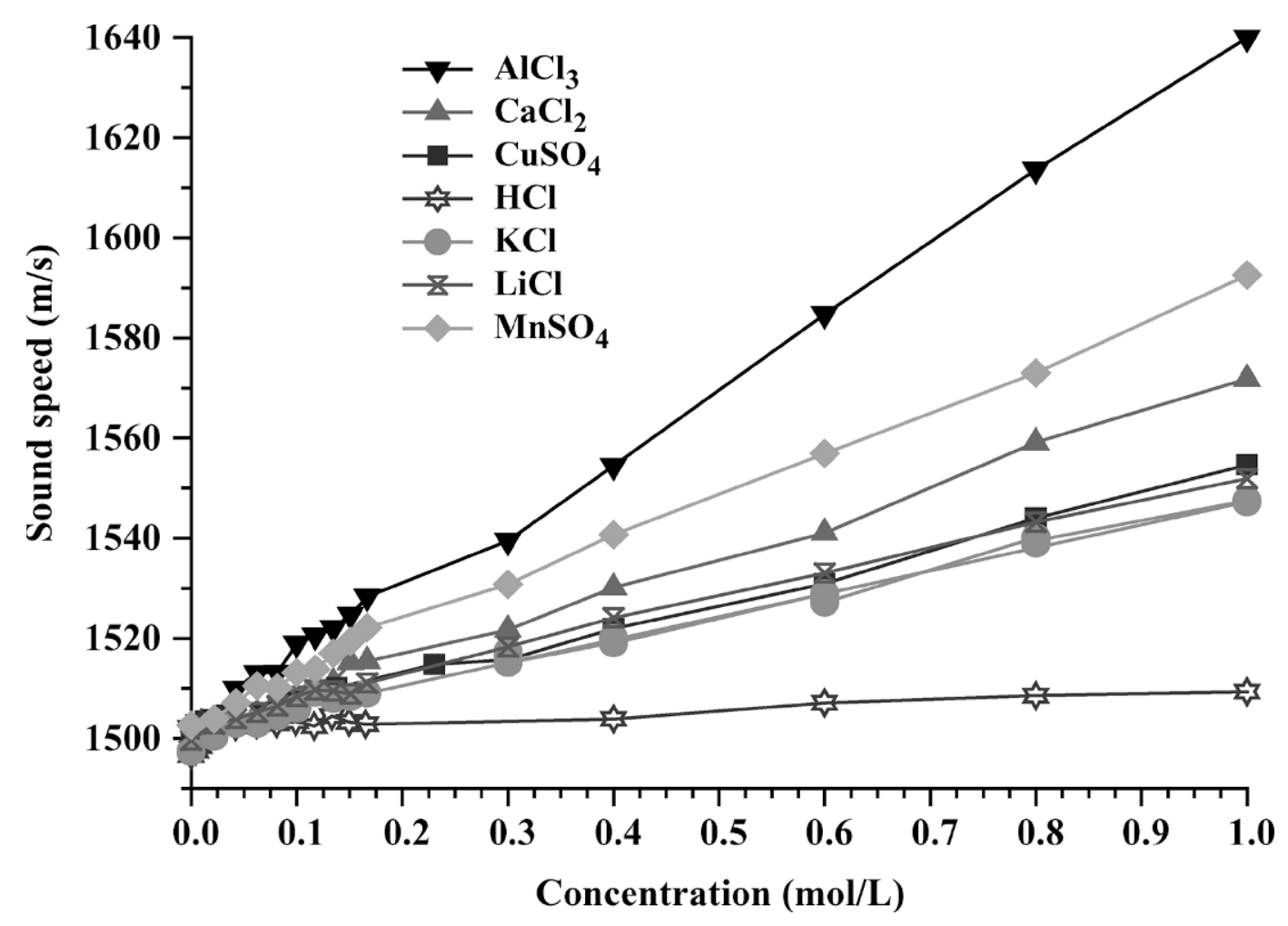

Figure 13. Sound speed of water versus concentration of various chemicals.

clude that it is possible to monitor variation of the water chemical composition with precision as small as 0.0001 $\mathrm{mol} / \mathrm{L}$.

Sound speed is also very sensitive to temperature. In water, it changes about $2.4 \mathrm{~m} / \mathrm{s}$ per degree Celsius.

In contrast, sound attenuation is much less sensitive to chemical composition. Figure 14 shows the attenuation spectra of water at $1 \mathrm{~mol} / \mathrm{L}$ concentration of similar chemical additives. It is seen that even at this very high concentration it changes significantly only for highly charged ions. This influence is much less pronounced at the lower concentrations that we might expect in food products. Figure 15 shows that even for a salt having 2:2 valence, the attenuation changes by only 0.1 $\mathrm{dB} / \mathrm{cm}$ per $\mathrm{MHz}$ with added salt concentration of 0.14 $\mathrm{mol} / \mathrm{L}$. It might be generally concluded that the impact of chemical variation on attenuation is negligible if concentration varies less than $0.1 \mathrm{~mol} / \mathrm{L}$.

Attenuation is not very sensitive to the molecular and ionic composition of the system, but it changes dramatically with the composition of the dispersed phase (Figure 3). In other words, the attenuation reflects changes occurring on the scale of colloid sizes, from nanometers to microns, whereas sound speed is more representative of effects that originate at a molecular scale of angstroms. This simple analysis indicates that sound speed is the better parameter for investigating effects on a molecular scale, whereas attenuation is more suitable for characterizing effects related to the heterogeneity and phase composition of the particular system.

\section{Ranges of Values}

The next point of interest is potential range of attenuation and sound speed in food products. Sound speed varies within the range of 1000 to $2000 \mathrm{~m} / \mathrm{s}$ for most food products. For dilute water-based food products, it would be close to $1500 \mathrm{~m} / \mathrm{s}$, which is the sound speed of water at room temperature.

The attenuation varies over a much wider dynamic range. That is why it is normally expressed on a logarithmic scale in decibels. Water has a very low attenuation compared with most other liquids. Its attenuation, expressed in units of $\mathrm{dB} / \mathrm{cm}$ per $\mathrm{MHz}$, increases linearly with frequency reaching approximately 0.2 at $100 \mathrm{MHz}$.

To be useful for a broad range of applications, an acoustic spectrometer must be able to cover a dynamic range from that of water up to at least $20 \mathrm{~dB} / \mathrm{cm}$ per $\mathrm{MHz}$ over a frequency range from 1 to $100 \mathrm{MHz}$. 


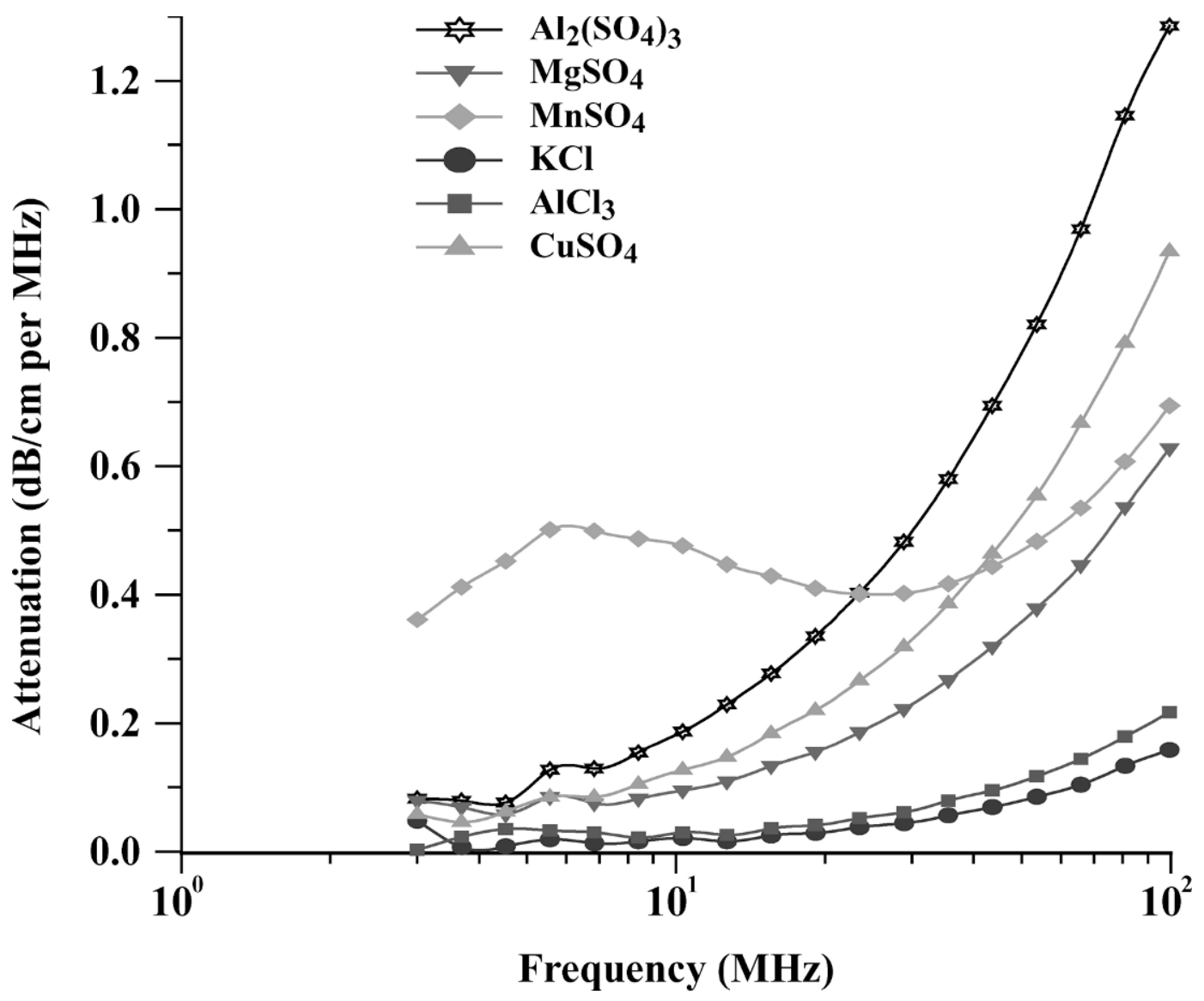

Figure 14. Attenuation of water versus concentration of various chemicals.

\section{Precision}

It is also possible to derive some conclusions regarding the required precision of the acoustic measurement because it depends directly on the precision of monitoring and maintaining other parameters. For instance, it is practically impossible to maintain a uniform temperature of the sample with a precision better than $0.01^{\circ} \mathrm{C}$. We know that a temperature change of $1^{\circ} \mathrm{C}$ leads to a sound speed change in water of $2.4 \mathrm{~m} / \mathrm{s}$. It follows that a variation of $0.01^{\circ} \mathrm{C}$ corresponds to a variation in sound speed of about $2.4 \mathrm{~cm} / \mathrm{s}$, or a fractional change of about $10^{-5}$. Obtaining precision much better than 1 part in $10^{-5}$ makes little sense because such precision would be masked by uncontrolled temperature variations.

It is possible to make another estimate of the meaningful precision of sound speed measurements from another viewpoint, the uncontrolled variation in chemical composition. It is known that maintaining dissolved ions and molecules composition in water with precision higher than $10^{-4} \mathrm{~mol} / \mathrm{L}$ is impossible. Adsorption of various chemical species from the atmosphere creates even larger uncontrolled variations. Figure 13 shows that sound speed changes about $100 \mathrm{~m} / \mathrm{s}$ for additions of 1 $\mathrm{mol} / \mathrm{L}$ of simple salts. It follows then that this uncontrolled variation in chemical composition leads to a change in sound speed of about $1 \mathrm{~cm} / \mathrm{s}$. It is interesting that the meaningful level of precision is the same from the viewpoint of uncontrolled temperature and from chemical composition.

Unfortunately, it is not possible to estimate the meaningful precision of attenuation measurement using a similar analysis. As we mentioned above, attenuation is less sensitive to chemical composition and temperature variations. The attenuation depends mostly on the composition and state of the dispersed phase. As a result, the precision of its measurement must be related somehow to the particles.

We have done this using resolution of the attenuation measurement for characterizing content of the large particles (Dukhin and Goetz, 2002). To resolve a single $1-\mu \mathrm{m}$ particle on the background of 100,000 particles with size $100 \mathrm{~nm}$, the attenuation must be measured with a precision of $0.01 \mathrm{~dB} / \mathrm{cm}$ per $\mathrm{MHz}$. Figure 4 shows that it is possible to measure attenuation with this level of precision for food products. 


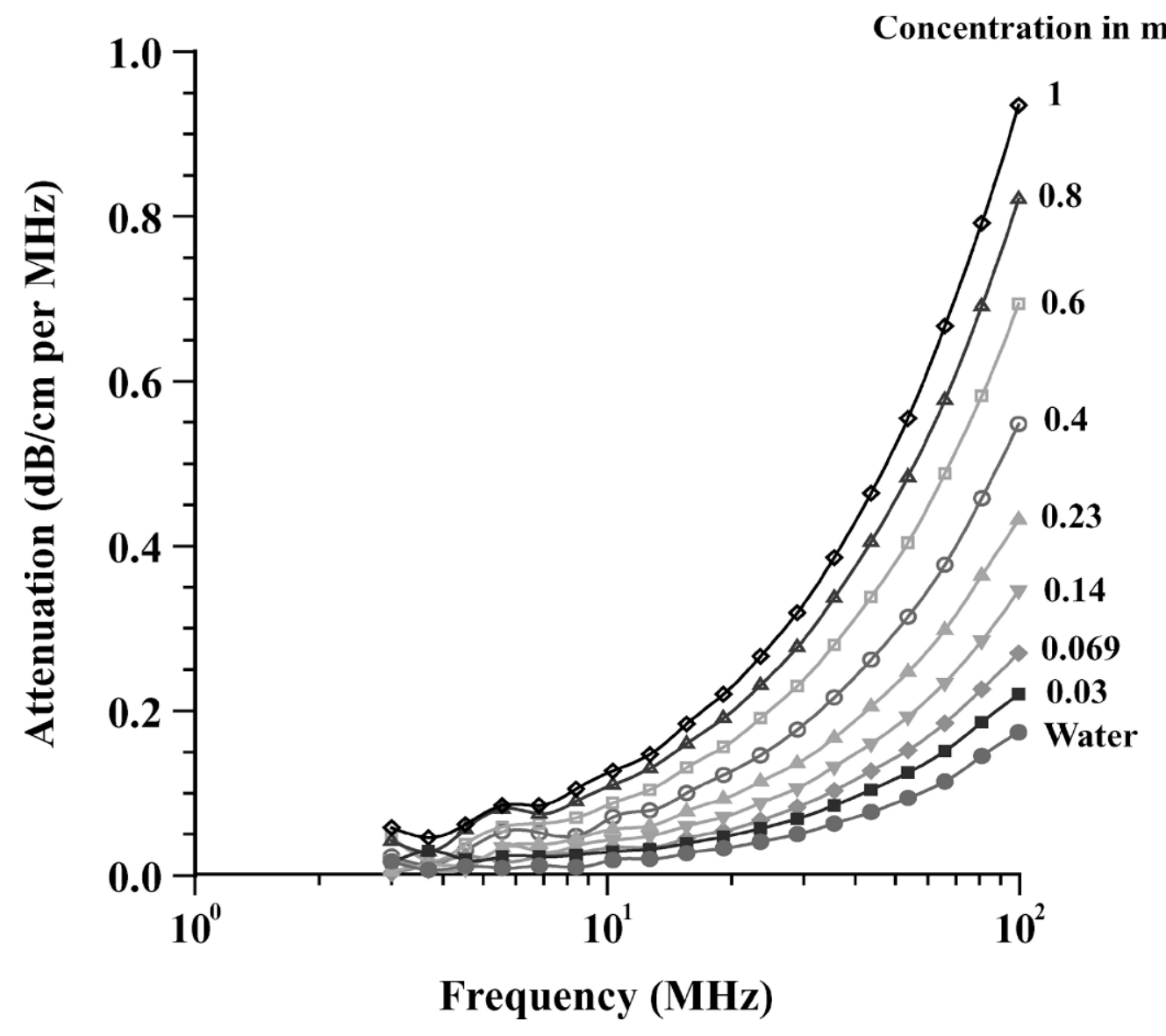

Figure 15. Attenuation of water versus concentration of $\mathrm{CuSO}_{4}$.

\section{Frequency Range}

The required frequency range depends on the purpose of the measurement. The wider the frequency range, the more information we obtain from which to calculate the droplet size distribution. Attenuation measurements over the frequency range from 1 to $100 \mathrm{MHz}$ makes it possible to characterize sizes as small as $5 \mathrm{~nm}$, as demonstrated by measurements of water-in-heptane microemulsions (Dukhin and Goetz, 2002). Restricting the frequency range to only the lower frequencies would dramatically limit the ability to measure very small particles as well as restrict the ability to characterize details of the particle size distribution.

At the same time, it should be noted that air bubbles in the sample may produce excess attenuation at low frequencies below $10 \mathrm{MHz}$. This might be especially true for some process control applications, where consistent removal of bubbles might present a problem. In such cases the particle size can still be computed using the higher frequency portion of the spectrum, with some loss in the ability to define some details of the distribution.
The selection of the most appropriate frequency is especially important for determining fat content, which requires an attenuation measurement at just one frequency. For accurate fat content determinations, the frequency should be chosen such that the intrinsic attenuation, which is independent of the size distribution, far exceeds the thermal attenuation, which in contrast is very size-dependent. Typically for dairy products, the optimum frequency would be in the range of 40 to $50 \mathrm{MHz}$.

\section{Appendix 2. Why Ultrasound Attenuates Propagation Through a Heterogeneous System}

There is a general formula describing the propagation of any energy field through a heterogeneous system: extinction $($ attenuation $)=$ scattering + absorption. According to Webster's dictionary, the verb "scatter" means "to separate and go in several directions," or "to reflect and refract in an irregular diffuse matter;" the noun "absorption" means "taking in and not reflecting." Applying these definitions to ultrasound, we can say that ultrasound is both scattered and absorbed when 
propagating through a heterogeneous system. Scattering by particles redirects the ultrasound energy away from the propagation path. Absorption, on the other hand, converts the ultrasound energy into heat due to interaction between the sound wave and both the particles and media.

There are several simplifications that are valid for many food products. First, submicron particles do not scatter ultrasound. They only absorb it. Second, the density of the particles in food products is usually very similar to the density of the liquid. This largely eliminates hydrodynamic effects. The propagation of ultrasound through food products is thus predominantly thermodynamic in nature.

It is quite easy to imagine the scattering of ultrasound; it is essentially the same as for light scattering. The first ideas of scattering were created by Lord Rayleigh for sound, not light (Rayleigh, 1896). It is more complicated to imagine absorption of ultrasound by particles and media. In order to simplify this effect, it has been split into 2 parts: absorption by particles and absorption by the liquid media.

Absorption by the liquid media is usually called "intrinsic attenuation." It occurs due to a variety of interactions on a molecular level. The attenuation spectra shown in Figures 14 and 15 are examples of the intrinsic attenuation of water with different added chemical species. Intrinsic attenuation is a measurable parameter. It is used as is, with no particular theoretical interpretation. The intrinsic attenuation of pure water, or some other pure liquid, can be used as a fingerprint. It is useful for testing instrument performance. For instance, Dispersion Technology Inc. instruments rely on water as testing sample. There is a direct relationship between the intrinsic attenuation and the viscosity of the liquid (Stokes, 1848).
Absorption of ultrasound by particles having a lowdensity contrast is usually called "thermal attenuation." It was first described almost 60 yr ago by Isakovich (1948). In cases where the density of the particle matches the density of the liquid, there is no relative particle-liquid motion. However, the compressibility of these soft particles is usually different than the compressibility of the surrounding liquid. As a result, the particle and the liquid expand to different degrees in the ultrasound field. This difference creates a tiny temperature gradient at the particle-liquid boundary. Energy flow at this boundary is irreversible according to thermodynamic principles, and it produces heat. Simply put, the presence of particles converts ultrasound energy to heat and the ultrasound is thereby attenuated. This is analogous to a refrigerator. It is known that refrigerators generate heat due to thermodynamic effect. We can model particles as tiny refrigerators that generate heat by taking energy from the ultrasound wave. This strange effect is measurable, and it is dependent on the thermodynamic properties of both the liquid and the particles. The relevant properties include the heat conductance, thermal expansion, and heat capacity, all determined at constant pressure. These parameters are known for many liquids. The thermal expansion coefficient is the most important of these, as the other 2 are similar for most liquids (Dukhin and Goetz, 2002). The thermal conductance is close to $1 \mathrm{~mW} / \mathrm{cm} \mathrm{K}$, whereas the heat capacity varies from 0.5 to 3 for most liquids. The thermal attenuation of a pure liquid is a function of these 3 properties. The total thermal attenuation of a dispersion is a function of the 3 thermal properties for each phase and also depends on the size distribution of the particles. A detailed theory of the thermal attenuation is provided in Dukhin and Goetz (2002). 